\title{
Features of criticality in precursory seismic electric signals and earthquakes in Greece
}

\author{
E. Dologlou \\ Solid State Section, Department of Physics, University of Athens, Panepistimiopolis, Zografou, 15784 Athens, Greece \\ Correspondence to: E. Dologlou (edologl@phys.uoa.gr)
}

Received: 26 February 2013 - Revised: 29 April 2013 - Accepted: 20 May 2013 - Published: 21 June 2013

\begin{abstract}
Very recently the recordings of precursory seismic electric signals (SESsf) in the island of Kōzu-shima, Japan, have been reported, and their interrelation with subsequent earthquakes was shown to be beyond chance. Almost simultaneously, the recording of SES activity in northern Greece was also reported, which was followed by a magnitude 5.7 earthquake in northern Greece. These facts strengthen the aspects on the precursory nature of SESs and also enable the updating of a previously found power law relation between the earthquake stress drop and the lead time of SESs. They led to an exponent of 0.330 , which falls in the range of critical exponents for fracture. The stability of this exponent, which results from a large amount of data during the last $30 \mathrm{yr}$, is remarkable and may thus confirm features of criticality in the pre-seismic region after the SES emission. The underlying physics are also discussed.
\end{abstract}

\section{Introduction}

Attempts to link earthquakes and critical phenomena are supported by recent findings that fractures in heterogeneous media exhibit critical behaviour (Lamaignere et al., 1996; Andersen et al., 1997; Sornette, 2000; Rundle et al., 2000). During the last preparatory stage of a large earthquake, the preseismic region in the earth's heterogeneous crust is characterized by the general feature of complex systems in a nearcritical state such as long-range correlations, fractal geometry and nonlinear dynamic processes (Telesca et al., 2005; Teotia and Kumar, 2011). A number of recent publications (Dologlou, 2008a, b, 2009, 2010, 2012a) showed an interconnection between dynamic parameters of large earthquakes and features of their precursory seismic electric signal, in terms of criticality. Seismic electric signals, termed as SESs, are found to precede large earthquake in Greece (Varotsos and Alexopoulos, 1984a, b; Varotsos et al., 1986, 1993) and Japan (Uyeda et al., 2009, 2002). SESs are detected by burring two electrodes at a depth of around two metres and a distance of some metres to few kilometres. They are recognized as transient low frequency $(\leq 1 \mathrm{~Hz})$ variations of the earth's telluric field and exhibit a series of properties such as the lead time and the selectivity effect. The lead time, which is time difference between the SES emission and the associated earthquake occurrence, can vary from some hours (for a single signal) to a few months (for SES activity). A better estimation (of the order of few days) of the time of the forthcoming earthquake has been recently achieved by means of a new time concept, termed natural time (Varotsos et al., 2002, 2006a, b, 2011; Sarlis et al., 2008), which instead of the conventional time evolution considers the order evolution of events (i.e. 1st, 2nd, etc). On the other hand the selectivity effect that reflects the ability of a SES station to be sensitive to some specific areas and remain inactive to others even at closer distances (Varotsos and Lazaridou, 1991; Varotsos et al., 1993) contributes to the determination of the epicentre of the impending earthquake.

A debate was organized by Geophysical Research Letters (Varotsos et al., 1996a, b) to answer the question of whether the success of the SES predictions can be attributed to chance. Very recently the following two facts have been almost simultaneously reported: first, in Japan, Orihara et al. (2012) showed that the interrelation between precursory SESs in the island of Kōzu-shima and their subsequent earthquakes was beyond chance, thus giving a clear answer to the aforementioned debate. Second, in Greece a SES activity was recorded in a station located in northern Greece (Varotsos et al., 2012) which was followed by a magnitude 5.7 earthquake in northern Aegean sea. This finding enables the examination 


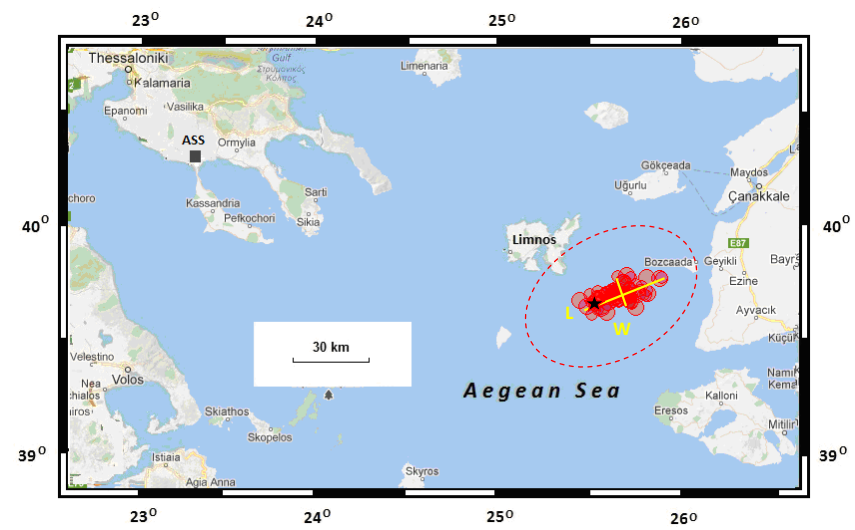

Fig. 1. Map of north-eastern Greece, with the distribution (forming a cluster-dashed ellipse) of all reported by EMSC aftershocks with $M_{\mathrm{W}} \geq 3$ (red circles) of the main shock on 8 January 2013 (event no. 22) and for the period 8 January 2013 (14:20 UTC) to 20 January 2013. The epicentre of the main shock (black star), the SES station ASS (solid square) and the aftershock area of length $L$ and width $W$ are depicted.

on whether the credibility of a power law relation with a critical exponent between the earthquake stress drop (which is the difference between the stress state at a point on a fault before and after the occurrence of the earthquake) and the SES lead time found by Dologlou (2009, 2010, 2012a, b) is preserved when these new data are introduced. This critical exponent based on a large amount of data of earthquakes and associated SESs is subjected to a continuous updating and seems to exhibit a remarkable stability and universality.

\section{Data and discussion}

Here we deal with the updating of this power law by using new data from 17 April 2012 (see Table 1 of Dologlou, 2012 b) till 20 January 2013. We again restrict ourselves to significant events $\left(M_{\mathrm{w}}>5.0\right)$. During this period only one SES was detected and one earthquake with $M_{\mathrm{w}}>5.0 \mathrm{oc}-$ curred in the broad region of Greece $\left(36-41^{\circ} \mathrm{N}, 19-26^{\circ} \mathrm{E}\right)$ (http://www.emsc-csem.org/Earthquake/europe/). Precisely, on 13 July 2012 a SES was recorded at ASS station close to Assyros village near Thessaloniki city (Varotsos et al., 2012) in northern Greece (Fig. 1 - solid square). This station had remained silent for the last $16 \mathrm{yr}$, and it is the only one which is found to be sensitive to earthquakes with epicentres in the north-eastern Greece $\left(39.2-41^{\circ} \mathrm{N}, 22.5-26^{\circ} \mathrm{E}\right)$. It is worth noting that also during these $16 \mathrm{yr}$ no large earthquake $(M \geq 5.5)$ occurred in the above area except the $M_{\mathrm{w}}=5.7$ event on 8 January 2013 at 14:16:08 UTC with epicentre at $39.65^{\circ} \mathrm{N}, 25.54^{\circ} \mathrm{E}$ (Fig. 1 - solid star) and seismic moment $M_{\mathrm{o}}=4.6 \times 10^{23} \mathrm{dyn} \mathrm{cm}$ (USGS).

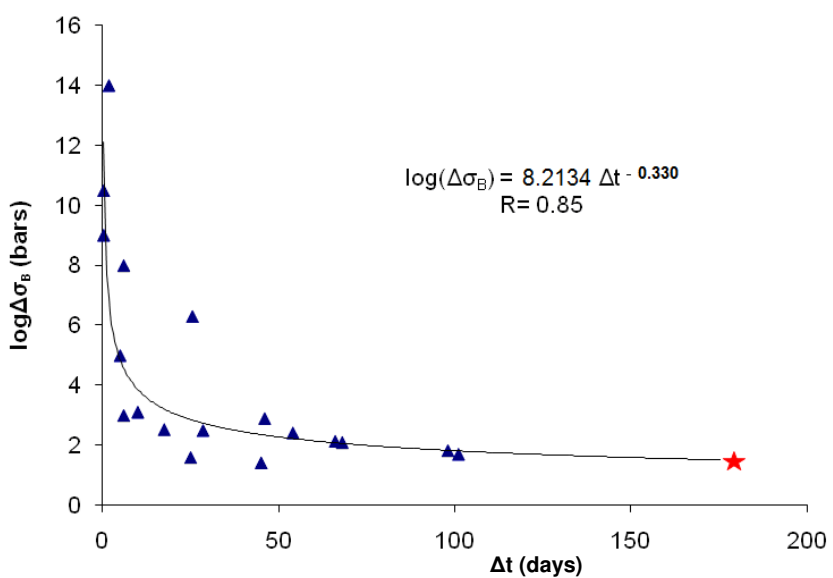

Fig. 2. The plot of the relation between the Brune's stress drop $\Delta \sigma_{B}$ and the lead time $\Delta t$ of all earthquakes listed in Table 1. Event nos. 11, 13 and 15 are excluded. The derived power law equation and the corresponding correlation coefficient $R$ are displayed at the top of the diagram. New data are marked by a red star.

In order to calculate the Brune's stress drop $\Delta \sigma_{B}$, we used the formula of Hanks and Wyss (1972):

$\Delta \sigma_{B}=0.44 M_{\mathrm{o}} / r^{3}$

where $r$ is the radius for a circular fault and it is estimated by applying the aftershock area technique (Kiratzi et al., 1985, 1991) as it is described in detail by Dologlou (2009). The aftershock area $S=\pi r^{2}$ with dimensions $L \times W=(36 \times$ 11) $\mathrm{km}^{2}$, where $L$ is the length and $W$ the width (Table 1 ), is defined from the cluster formed by the distribution of the epicentres of the aftershock sequence (Fig. 1 - dashed ellipse). We consider as aftershocks all the events reported by the European Mediterranean Seismological Center, EMSC (http://www.emsc-csem.org), with $M_{\mathrm{w}}>3.0$ from 8 January 2013 to 20 January 2013 and in the area $39-40^{\circ} \mathrm{N}$, $25-26.2^{\circ} \mathrm{E}$. The computed stress drop is found as $\Delta \sigma_{B}=$ 1.43 bars (Table 1) while the lead time $\Delta t=179$ days.

We now insert the above values into the latest reported power law relation $\Delta \sigma_{B}=8.195 \Delta t^{-0.329}$ (Dologlou, 2012b), which is based on data from 1981 to 2012 (Table 2 - event nos. 1-21), and it is subjected to a continuous updating. We note that event nos. 11, 13 and 15 are always excluded from this relation for reasons explained in detail (Dologlou et al., 2008; Dologlou, 2008a, 2009). The new derived exponent value is $\alpha=0.330 \pm 0.01$ (Fig. 2). In order to check the contribution of this new data point (no. 22), we recalculate the power law by considering, instead of the last event no. 22 , one of the events no. 11 , no. 13 and no. 15 each time, and we compare the derived values with the previously computed one ( $\alpha=0.329 \pm 0.01$ with correlation coefficient $R=0.85$; see Fig. 3 ) from the existing 18 points listed in Table 1 . 
Table 1. The USGS reported date and magnitude of the main shock along with its seismic moment, range of values in the dimensions of the aftershock area $L$ and $W$, corresponding calculated values for $\Delta \sigma_{B}$, the critical exponent $\alpha$ and the lead time $\Delta t$. Mean values are also given.

\begin{tabular}{|c|c|c|c|c|c|c|c|c|}
\hline no. & $\begin{array}{l}\text { Date } \\
\text { yy mm dd }\end{array}$ & $M_{\mathrm{W}}$ & $\begin{array}{l}M_{\mathrm{O}} \\
\left(10^{23} \text { dyn cm }\right)\end{array}$ & $\begin{array}{l}L \\
(\mathrm{~km})\end{array}$ & $\begin{array}{l}W \\
(\mathrm{~km})\end{array}$ & $\begin{array}{l}\Delta \sigma_{B} \\
\text { (bars) }\end{array}$ & $\alpha$ & $\begin{array}{l}\Delta t \\
\text { days }\end{array}$ \\
\hline \multirow[t]{2}{*}{22} & 130108 & 5.7 & 4.6 & $35-37$ & $10-12$ & $1.72-1.21$ & $0.323-0.337$ & 179 \\
\hline & Mean value & & & 36 & 11 & 1.43 & $0.330 \pm 0.01$ & \\
\hline
\end{tabular}

Table 2. The updated version of the table reported by Dologlou (2010, 2012a, b) included the new earthquake on 8 January 2013 . All 22 earthquakes with available stress drop values and precursory SES signals in Greece from 1981 to 2013, along with their dates, epicentres, depths, moment magnitudes $M_{\mathrm{W}}$, stress drop $\Delta \sigma_{\mathrm{B}}$ and $\Delta t$ SES lead times are given.

\begin{tabular}{|c|c|c|c|c|c|c|c|c|c|c|}
\hline no. & yy mm dd & h & $\mathrm{m}$ & $\mathrm{s}$ & lat & long & $d(\mathrm{~km})$ & $M_{\mathrm{W}}$ & $\Delta \sigma_{B}$ (bars) & $\Delta t$ (days) \\
\hline 1 & 811219 & 14 & 10 & 50.7 & 39.24 & 25.23 & 12 & 6.8 & 9.01 & 0.3 \\
\hline 2 & 820118 & 19 & 27 & 24.5 & 40.00 & 24.32 & 6 & 6.6 & 10.5 & 0.3 \\
\hline 3 & 830117 & 12 & 41 & 29.0 & 38.09 & 20.19 & 10 & 6.9 & 14.0 & 1.8 \\
\hline 4 & 860913 & 17 & 24 & 34.0 & 37.03 & 22.20 & 15 & 5.9 & 5.0 & 5 \\
\hline 5 & 881016 & 12 & 34 & 06.0 & 37.95 & 20.90 & 29 & 5.9 & 2.53 & 17.5 \\
\hline 6 & 950504 & 00 & 34 & 11.0 & 40.54 & 23.63 & 15 & 5.4 & 2.5 & 28.5 \\
\hline 7 & 950513 & 08 & 47 & 15.0 & 40.16 & 21.67 & 15 & 6.5 & 6.3 & 25.5 \\
\hline 8 & 950615 & 00 & 15 & 56.0 & 38.10 & 22.46 & 15 & 6.5 & 2.9 & 46 \\
\hline 9 & 971118 & 14 & 07 & 53.0 & 37.33 & 20.84 & 23 & 6.6 & 1.42 & 45 \\
\hline 10 & 990907 & 11 & 56 & 56.0 & 37.97 & 23.60 & 15 & 6.0 & 3.0 & 6 \\
\hline $11^{*}$ & 010726 & 00 & 21 & 44.0 & 38.96 & 24.29 & 15 & 6.5 & 9.0 & 130 \\
\hline 12 & 030814 & 05 & 15 & 08.0 & 38.70 & 20.67 & 15 & 6.3 & 8.0 & 6 \\
\hline $13^{*}$ & 080106 & 05 & 14 & 20.0 & 37.22 & 22.69 & 75 & 6.2 & 9.0 & 60 \\
\hline 14 & 080204 & 20 & 25 & 09.5 & 38.08 & 21.94 & 20 & 5.0 & 1.60 & 25 \\
\hline $15^{*}$ & 080214 & 10 & 09 & 22.7 & 36.50 & 21.67 & 29 & 6.9 & 9.0 & 30 \\
\hline 16 & 080608 & 12 & 25 & 29.7 & 37.96 & 21.52 & 16 & 6.3 & 1.83 & 98 \\
\hline 17 & 090216 & 23 & 16 & 38.5 & 37.13 & 20.78 & 15 & 5.5 & 2.14 & 66 \\
\hline 18 & 091103 & 05 & 25 & 08.1 & 37.50 & 20.49 & 10 & 5.8 & 3.11 & 10 \\
\hline 19 & 100118 & 15 & 56 & 09.0 & 38.40 & 21.96 & 1 & 5.5 & 2.1 & 68 \\
\hline 20 & 110719 & 07 & 13 & 12.2 & 37.21 & 19.92 & 9 & 5.1 & 2.42 & 54 \\
\hline 21 & 120416 & 11 & 23 & 43.2 & 36.63 & 21.48 & 29 & 5.8 & 1.71 & 101 \\
\hline 22 & 130108 & 14 & 16 & 08.0 & 39.66 & 25.54 & 10 & 5.7 & 1.43 & 179 \\
\hline
\end{tabular}

* Events 11, 13 and 15 are excluded from the power law relation for reasons explained in Dologlou, 2008c, a and 2009, respectively. (a) for data references for events 1-19 (see Table 1 of Dologlou, 2010 and references therein); (b) for data references for events 20 and 21 (see Table 1 of Dologlou, 2012a and Dologlou, 2012b, respectively).

Thus for event no. 11, the derived value $\alpha=0.269 \pm 0.01$ with correlation coefficient $R=0.67$ (see Fig. 4a) significantly deviates (i.e. outside of the experimental error) from the above value of the 18 points (i.e. $\alpha=0.329 \pm 0.01$ ). Similarly, by using event no. 13 we get the value $\alpha=$ $0.294 \pm 0.01$ with $R=0.72$ (Fig. 4b), which again lies outside the expected error. Finally, by introducing event no. 15 we get $\alpha=0.313 \pm 0.01$ and $R=0.76$ (Fig. 4c), which also falls outside the error range. In other words while the no. 22 event gives a value of the exponent $\alpha=0.330 \pm 0.01$ (Fig. 2), which is in excellent agreement with the previously found one $\alpha=0.329$ (Dologlou, 2012b), the addition of any of the other three points no. 11 , no. 13 and no. 15 markedly deviates from the behaviour of the preceding 18 events. Hence, the introduction of the new point (i.e. no. 22) shows that the exponent exhibits a remarkable stability and strengthens our previous results. Its value falls within the range (i.e. 0.3-0.4) of critical exponents for fracture (Surkov et al., 2002) and thus unveil signatures of criticality in the dynamic procedures that govern the candidate seismic region.

Various possible physical mechanisms of generating earthquake-related EM signals, such as the electrokinetic effect (Mizutani et al., 1976; Johnston, 1997) and the piezoelectric effect (Nitsan, 1977; Ikeya et al., 1997; Huang, 2002, 2011), have been proposed. In particular we mention a recent work considering the electrokinetic effect as a mechanism causing the coupling between seismic and electromagnetic energies (Ren et al., 2012), and the electromagnetic fault model taking into account the piezoelectric effect and 


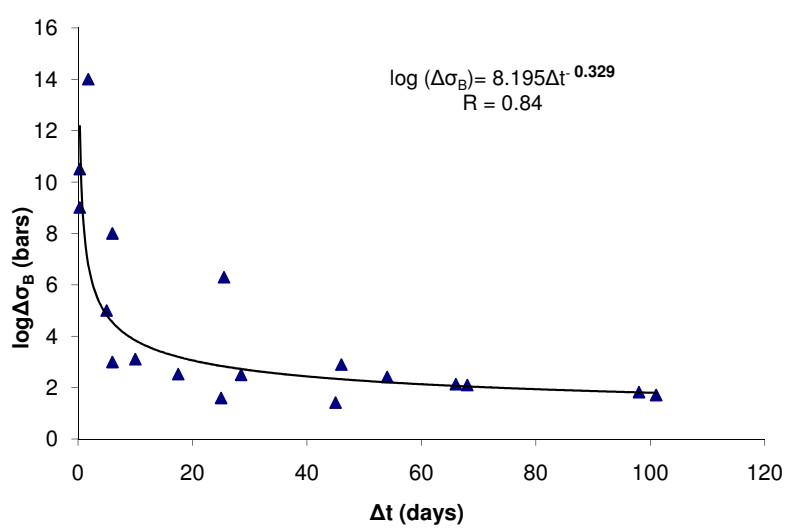

Fig. 3. The plot of the relation between the Brune's stress drop $\Delta \sigma_{B}$ and the lead time $\Delta t$ as reported by Dologlou (2012b) (i.e. all events of Table 1 except event nos. 11, 1315 and 22). The derived power law equation and the corresponding correlation coefficient $R$ are displayed at the top of the diagram.

the stress drop due to dislocation theory of a fault (Huang, 2002, 2011).

Here, we rely on an alternative model for the generation of earthquake-related very low frequency EM signals, which is based on the stress-stimulated currents (Varotsos, 2005), and it is briefly discussed below.

When the increasing tectonic stress in the pre-seismic area reaches a critical value, the dipoles that are formed by aliovalent impurities in the solids (Varotsos and Alexopoulos, 1977, 1981), which are included in the rocks, change cooperatively orientation, thus giving rise to a transient electric signal which constitutes the SES (Varotsos and Alexopoulos, 1984c). The emission of the SES implies the entrance of the candidate seismic region into the last preparatory stage of the forthcoming earthquake where nonlinear dynamic processes expressed by power law relations with critical exponents (Sornette et al., 1989; Sornette and Sammis, 1995) prevail. Additionally, critical features are recognized through a power law relation (Varotsos and Alexopoulos, 1984a; see p. 91), which interconnects the magnitude $M$ of the impending earthquake and the amplitude $E$ of the associated SES as

$\log E=a M+b$,

where $a \approx 0.3--0.4$ and $b$ is a constant depending on the geoelectrical structure in the surroundings of the station. We note that although the presence of power law relations is a necessary condition, it is not sufficient to confirm a critical stage. For example, both artificial noise and SES activities exhibit power law behaviour, but only SESs are characterized by critical dynamics (Varotsos et al., 2011).

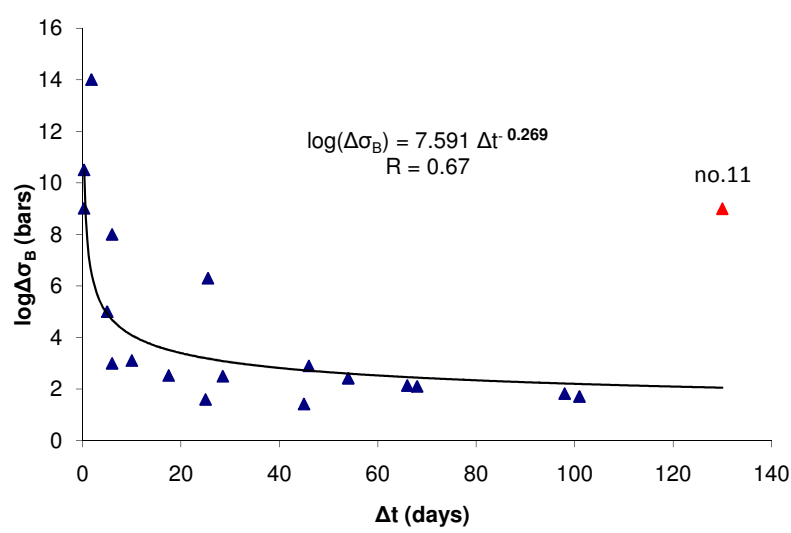

$\mathbf{a}$

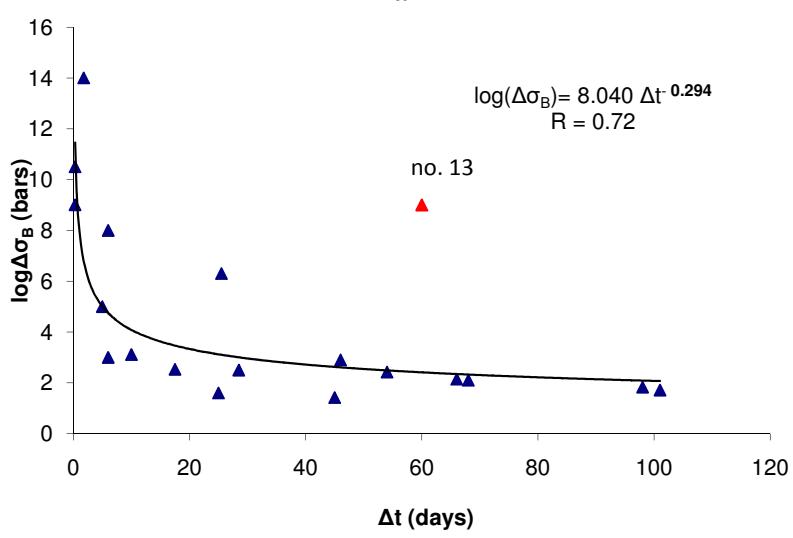

b

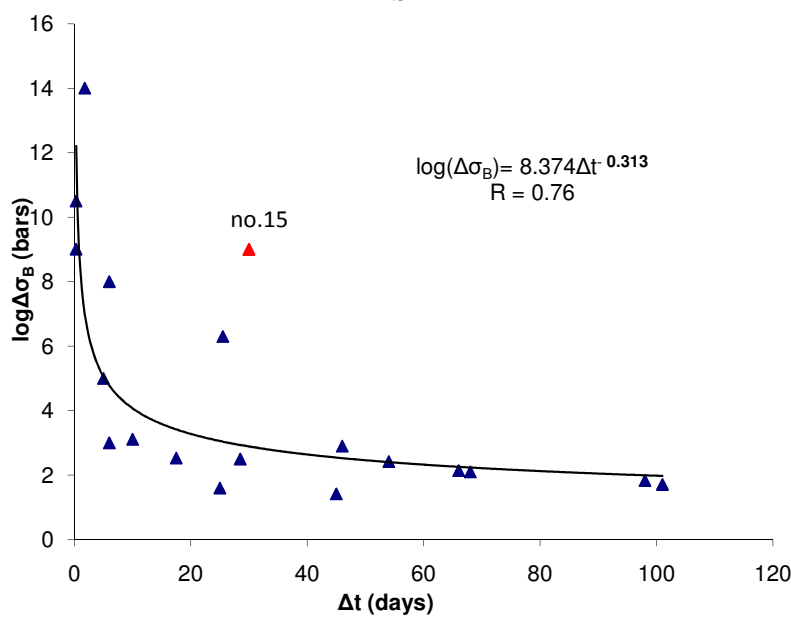

c

Fig. 4. The plot of the relation between the Brune's stress drop $\Delta \sigma_{B}$ and the lead time $\Delta t$ of all earthquakes listed in Table 1. (a) With included event no. 11 (red triangle) and excluded nos. 13, 15 and 22. (b) With included event no. 13 (red triangle) and excluded nos. 11, 15 and 22. (c) With included event no. 15 (red triangle) and excluded nos. 11, 13 and 22. The derived power law equation and the corresponding correlation coefficient $R$ are displayed at the top of each diagram. 


\section{Conclusions}

The insertion of new latest data in a previously found power law relation which interconnects the earthquake stress drop and the SES lead time allows the following two conclusions: first, in Japan the suggestion that the interrelation between precursory SESs in the island of Kōzu-shima and their subsequent earthquakes was beyond chance is strengthened. Second, the new derived value 0.330 of the critical exponent in the above relation falls in the range values of critical exponents for fracture, and it is in excellent accord with the last one (i.e. 0.329) reported by the author. The stability of this exponent resulting from a large amount of data during the last 30 years is remarkable and may suggest features of criticality in the pre-seismic region after the SES emission, which are also observed in places other than the eastern Mediterranean geotectonic block such as Japan, north-eastern Asia. The underlying physics are also discussed.

Edited by: L. Telesca

Reviewed by: two anonymous referees

\section{References}

Andersen, J. V., Sornette, D., and Leung, K.-T.: Tri-critical behavior in rupture induced by disorder, Phys. Rev. Lett., 78, 2140-2143, 1997.

Dologlou, E.: Power law relationship between parameters of earthquakes and precursory electrical phenomena, Nat. Hazards Earth Syst. Sci., 8, 977-983, doi:10.5194/nhess-8-977-2008, 2008a.

Dologlou, E.: Possible relationship between Seismic Electric Signals (SES) lead time and earthquake stress drop, Proc. Jpn. Acad., Ser. B, 84, 117-122, doi:10.2183/pjab.84.117, 2008b.

Dologlou, E.: Power law relationship between parameters of earthquakes and precursory electrical phenomena revisited, Nat. Hazards Earth Syst. Sci., 9, 17-23, doi:10.5194/nhess-9-17-2009, 2009.

Dologlou, E.: Power law relationship between parameters of earthquakes and precursory electrical phenomena revisited II, Nat. Hazards Earth Syst. Sci., 10, 1403-1409, doi:10.5194/nhess-101403-2010, 2010.

Dologlou, E.: Stability of a power law relation between characteristics of earthquakes and electric precursors, Nat. Hazards Earth Syst. Sci., 12, 1783-1787, doi:10.5194/nhess-12-17832012, 2012a.

Dologlou, E.: Testing the critical exponent in the relation between stress drop of earthquake and lead time of seismic electric signal, Nat. Hazards Earth Syst. Sci., 12, 2603-2607, doi:10.5194/nhess-12-2603-2012, 2012b.

Dologlou, E., Hadjicontis, V., and Mavromatou, C.: Electrical precursors of earthquakes in Aegean Sea during the last decade (1997-2007), Nat. Hazards Earth Syst. Sci., 8, 123-128, doi:10.5194/nhess-8-123-2008, 2008.

Hanks, T. and Wyss, M.: The use of body wave spectra in the determination of seismic source parameters, B. Seismol. Soc. Am., 62, 561-589, 1972.
Huang, Q.: One possible generation mechanism of co-seismic electric signals, Proc. Jpn Acad. B, 78, 173-178, 2002.

Huang, Q.: Rethinking earthquake-related DC-ULF electromagnetic phenomena: towards a physics-based approach, Nat. Hazards Earth Syst. Sci., 11, 2941-2949, doi:10.5194/nhess-112941-2011, 2011.

Ikeya, M., Takaki, S., Matsumoto, H., Tani, A., and Komatsu, T.: Pulsed charge model of fault behavior producing seismic electric signals (SES), J. Circuits Syst. Comput., 7, 153-164, doi:10.1142/S0218126697000115, 1997.

Johnston, M. J. S.: Review of electric and magnetic fields accompanying seismic and volcanic activity, Surv. Geophys., 18, 441475, 1997.

Kiratzi, A. A., Karakaisis, G. F., Papadimitriou, E. E., and Papazachos, B. C.: Seismic-Source Parameter Relations for Earthquakes in Greece, Pure Appl. Geophys., 123, 27-41, 1985.

Kiratzi, A. A., Wagner, G. S., and Langston, C. A.: Source Parameters of Some Large Earthquakes in Nortern Aegean Determined by Body Waveform Inversion, Pure Appl. Geophys., 135, 515527, 1991.

Lamaignère, L., Carmona, F., and Sornette, D.: Experimental realization of critic thermal fuse rupture, Phys. Rev. Lett., 77, 2738 2741, 1996.

Mizutani, H., Ishido, T., Yokokura, T., and Ohnishi, S.: Electrokineticphenomena associated with earthquake, Geophys. Res. Lett., 3, 365-368, 1976.

Nitsan, U.: Electromagnetic emission accompanying fracture of quartz-bearing rocks, Geophys. Res. Lett., 4, 333-336, 1977.

Orihara, Y., Kamogawa, M., Nagao, T., and Uyeda, S.: Preseismic anomalous telluric current signals observed in Kozu-shima Island, Japan, P. Natl. Acad. Sci. USA, 109, 19125-19128, doi:10.1073/pnas.1215669109, 2012.

Ren, X., Chen, X., and Huang, Q.: Numerical simulation of coseismic electromagnetic fields associated with seismic waves due to finite faulting in porous media, Geophys. J. Int., 188, 925-944, doi:10.1111/j.1365-246X.2011.05309.x, 2012.

Rundle, B. J., Turcotte, D. L., and Klein, W.: Geocomplexity and the Physics of Earthquakes, Am. Geophys. Union, Washington DC, 2000.

Sarlis, N. V., Skordas, E. S., Lazaridou, M. S., and Varotsos, P. A.: Investigation of the seismicity after the initiation of a Seismic Electric Signal activity until the main shock, Proc. Jpn. Acad., Ser. B, 84, 331-343, 2008.

Sornette, D. (Ed.): Critical Phenomena in Natural Sciences Chaos, Fractals, Self organization and Disorder: Concepts and Tools, Springer Ser. Synerg., Heidelberg, Springer-Verlag, 2000.

Sornette, D. and Sammis, C. G.: Complex critical exponents from renormalization group theory of earthquakes: Implications for earthquake predictions, J. Phys. I., 5, 607-619, doi:10.1051/jp1:1995154, 1995.

Sornette, D., Lagier, M., Roux, S., and Hansen, A.: Critical piezoelectricity in percolation, J. Phys. France, 50, 2201-2216, 1989.

Surkov, V., Uyeda, S., Tanaka, H., and Hayakawa, M.: Fractal Properties of medium and seismoelectric phenomena, J. Geodyn., 33, 477-487, doi:10.1016/S0264-3707(02)00009-1, 2002.

Telesca, L., Lapenna, V., and Macchiato, M.: Multifractal fluctuations in seismic interspike series, Physica A, 354, 629-640, doi:10.1016/j.physa.2005.02.053, 2005. 
Teotia, S. S. and Kumar, D.: Role of multifractal analysis in understanding the preparation zone for large size earthquake in the North-Western Himalaya region, Nonlin. Processes Geophys., 18, 111-118, doi:10.5194/npg-18-111-2011, 2011.

Uyeda, S., Kamogawa, M., and Tanaka H.: Analysis of electrical activity and seismicity in the natural time domain for the volcanicseismic swarm activity in 2000 in the Izu Island region, Japan, J. Geophys. Res., 114, B02310, doi:10.1029/2007JB005332, 2009.

Uyeda, S., Hayakawa, M., Nagao, T., Molchanov, O., Hattori, K., Orihara, Y., Gotoh, K., Akinaga, Y., and Tanaka, H.: Electric and magnetic phenomena observed before the volcano-seismic activity in 2000 in the Izu Island Region, Japan, P. Natl. Acad. Sci. USA, 99, 7352-7355, doi:10.1073/pnas.072208499, 2002.

Varotsos, P. A. (Ed): The Physics of Seismic Electric Signals, TerraPub, Tokyo, 2005.

Varotsos, P. and Alexopoulos, K.: Calculation of the formation entropy of vacancies due to anharmonic effects, Phys. Rev. B, 15, 4111-4114, doi:10.1103/PhysRevB.15.4111, 1977.

Varotsos, P. and Alexopoulos, K.: Decisive importance of the bulk modulus and the anharmonicity in the calculation of migration and formation volumes, Phys. Rev. B, 24, 904-910, doi:10.1103/PhysRevB.24.904, 1981.

Varotsos, P. and Alexopoulos, K.: Physical properties of the variations of the electric field of the earth preceding earthquakes, I, Tectonophysics, 110, 73-98, 1984a.

Varotsos, P. and Alexopoulos, K.: Physical properties of the variations of the electric field of the earth preceding earthquakes, II. Determination of epicentre and magnitude, Tectonophysics, 110 , 99-125, 1984b.

Varotsos, P. and Alexopoulos, K.: Connection between the formation volume and formation Gibbs energy in noble gas solids, Phys. Rev. B, 30, 7305-7306, doi:10.1103/PhysRevB.30.7305, 1984c.

Varotsos, P. and Lazaridou, M.: Latest aspects of earthquake Prediction in Greece based on Seismic Electric Signals. I, Tectonophysics, 188, 321-347, 1991.
Varotsos, P., Alexopoulos, K., Nomicos, K., and Lazaridou, M.: Earthquake prediction and electric signals, Nature, 322, p. 120, doi:10.1038/322120a0, 1986.

Varotsos, P., Alexopoulos, K., and Lazaridou M.: Latest aspects of earthquake prediction in Greece based on Seismic Electric Signals II, Tectonophysics, 224, 1-37, 1993.

Varotsos, P., Eftaxias, K., Vallianatos, F., and Lazaridou M.: Basic principles for evaluating an earthquake prediction method, Geophys. Res. Lett., 23,1295-1298, doi:10.1029/96GL00905, 1996a.

Varotsos, P., Eftaxias, K., Lazaridou M., Antonopoulos, G., Makris, J., and Poliyiannakis, J.: Summary of five principles suggested by P. Varotsos et al. (1996) and the additional questions raised in this debate, Geophys. Res. Lett., 23, 1449-1452, doi:10.1029/96GL01437, 1996b.

Varotsos, P., Sarlis, N. V., and Skordas, E. S.: Long-range correlations in the electric signals that precede rupture, Phys. Rev. E, 66, 011902, doi:10.1103/PhysRevE.66.011902, 2002.

Varotsos, P., Sarlis, N., Skordas, E., Tanaka, H., and Lazaridou, M.: Entropy of seismic electric signals: analysis in natural time under time reversal, Phys. Rev. E, 73, 031114, doi:10.1103/PhysRevE.73.031114, 2006a.

Varotsos, P., Sarlis, N., Skordas, E., Tanaka, H., and Lazaridou, M.: Attempt to distinguish long-range temporal correlations from the statistics of the increments by natural time analysis, Phys. Rev. E, 74, 021123, doi:10.1103/PhysRevE.74.021123, 2006b.

Varotsos, P., Sarlis, N. V., and Skordas, E. S. (Eds): Natural Time Aanlysis: The new view of time Precursory Seismic Electric Signals, Earthquakes and other Complex Time-Series, SpringerVerlag, Heidelberg, 2011.

Varotsos, P. A., Sarlis, N. V., and Skordas, E. S.: Order parameter fluctuations in natural time and $b$-value variation before large earthquakes, Nat. Hazards Earth Syst. Sci., 12, 3473-3481, doi:10.5194/nhess-12-3473-2012, 2012. 